\title{
Use of FOIA by animal rights activists
}

\author{
B. Taylor Bennett, Andrew D. Cardon \& Matthew R. Bailey
}

The federal Freedom of Information Act $^{1}$, commonly known as FOIA, governs access to records in the possession of federal agencies, such as the United States Department of Agriculture (USDA), National Institutes of Health (NIH), the Federal Drug Administration and the Centers for Disease Control. It was originally enacted in 1966 in order to promote transparency and ensure accountability. However, FOIA can also be exploited by outside groups to acquire information with the intention of spreading propaganda and curtailing progress in activities with which they disagree. Animal rights activists have increasingly turned to FOIA to acquire information about biomedical research in order to request investigations, to seek enforcement actions for alleged issues involving noncompliance and to call for increased fines. The National Association for Biomedical Research regularly monitors incoming FOIA requests at both the USDA and NIH. These requests are reviewed to identify the submitter, the submitter's affiliation and the specific nature of the request. In recent years, animal rights activists have been submitting broadly worded FOIA requests, in some cases seeking information about more than 25 research institutions in a single request. This not only increases the amount of information obtained, it also serves to obscure the identity of a targeted institution by making it one of many named in a request. The financial costs to government agencies to provide this type of information are considerable and in fiscal year 2014, direct FOIA expenses were nearly $\$ 462$ million. At the Animal and Plant Health Inspection Service (APHIS) and the NIH alone, 44 individuals were counted as "full-time FOIA staff" and FOIA costs exceeded \$5 million.
A closer analysis of FOIA logs for fiscal year 2014 shows that APHIS received 919 requests for information. Of these FOIA requests 215 (approximately 24\%) were identified as being submitted by animal rights or animal interest organizations or by individuals that appeared to be associated with such groups. Because some of the requests were broadly worded, the number of requests alone does not necessarily correspond to the volume of information released. Animal rights organizations most frequently requested information about exhibitors, actions related to horses and wildlife, dealers and research facilities. Requests for information about complaints and enforcement activity taken by APHIS were also frequently sought. Of the 215 requests identified as coming from animal rights groups, 40 were directly related to research facilities. These requests were made both by individuals and representatives of national animal rights groups, and they typically sought information regarding APHIS inspections. These requests were often broadly worded and seeking inspection reports as well as any supporting documentation, such as photographs, videos and reproduced documents.

During 2014 the NIH received a total of 1,173 FOIA requests, with 94 (approximately $8 \%$ ) of the requests submitted by animal rights or animal interest organizations or by individuals associated with such groups. All of the requests filed by animal rights groups to the NIH sought information related to research and research organizations. As with requests submitted to APHIS, it is important to note that the number of requests alone does not necessarily correspond to the volume of information released. Some requests were for individual documents, while others were broadly worded and encompassing hundreds or even thousands of pages of records associated with numerous research institutions. Animal rights organizations most frequently requested information related to specific grant applications and often included requests for summary statements, progress reports and correspondence pertaining to grants. Requests for all correspondence between specific research institutions and the Office of Laboratory Animal Welfare (OLAW) increased during 2014 as well. OLAW received a total of 27 requests for information on 217 institutions compared to 12 requests involving 28 institutions in 2013.

FOIA was originally enacted to ensure government accountability and transparency, but it can also be abused by organizations and individuals and add significant costs to the biomedical research process. Animal rights organizations continue to use FOIA to obtain information from APHIS and the NIH about research institutions, adding to the cost of more than $\$ 5$ million in taxpayer money in fiscal year 2014 that is needed to respond to FOIA requests. Because of the broad nature of the requests from animal rights activists, it is likely that these requests cost agencies more than average FOIA requests. Based on an early analysis of fiscal year 2015 data, activists continue to exploit FOIA by submitting broad requests for large amounts of data about research facilities. Research facilities should carefully review any information submitted to a federal agency to ensure that it accurately reflects the institution's animal care and use program and does not contain proprietary or personal information. Institutions should always be cognizant that information submitted to the federal government can be released at any time.

1. Freedom of Information Act, 5 USC, Section 552, 2006. 